\title{
Nutritional Deficiencies, Bariatric Surgery, and Serum Homocysteine Level: Review of Current Literature
}

\author{
Natalia Komorniak $^{1} \cdot$ Małgorzata Szczuko ${ }^{1}$ (D) $\cdot$ Bartosz Kowalewski ${ }^{2} \cdot$ Ewa Stachowska $^{1}$
}

Published online: 31 August 2019

(C) The Author(s) 2019

\begin{abstract}
Obesity is currently one of the biggest global health problems. In the case of severe obesity, bariatric surgeries are considered to be the most important method of treatment. The 2 most commonly performed bariatric surgery procedures include Roux-en-Y gastric bypass and sleeve gastrectomy. However, these methods are not free from complications, and the most common ones (moderately long or long term) are micronutrient deficiencies. The deficiency of vitamins B6, B12, and folic acid as cofactors of the folate cycle contributes to the development of hyperhomocysteinemia. It seems that apart from nutritional factors, there are other aspects that have a significant influence on the concentration of homocysteine in blood, such as the type of conducted bariatric surgery, the post-surgical concentration of betaine and creatinine, and the clearance of methionine (i.e., the mutations of the gene that encodes the MTHFR reductase as well as other genes associated with the process of methylation, e.g., methionine synthase). Their presence might be one of the causes of the increased concentration of homocysteine after surgery despite the fact that patients take vitamin-mineral supplementation.
\end{abstract}

Keywords Homocysteine $\cdot$ Bariatric surgery $\cdot$ MTHFR $\cdot$ Nutritional deficiencies $\cdot$ Obesity

\section{Introduction}

Obesity is currently one of the biggest global health problems [1]. Its frequency of occurrence tripled since 1975, reaching 650 million obese adults in 2016 [2]. The prognosis based on the present increasing tendency indicates that by 2030 , there will be 65 million more obese patients in the USA and 11 million more in the UK, which will result in the increase in

Małgorzata Szczuko

malgorzata.szczuko@pum.edu.pl

Natalia Komorniak

norma155@wp.pl

Bartosz Kowalewski

bartek.kowalewski@wp.pl

Ewa Stachowska

ewast@pum.edu.pl

1 Department of Human Nutrition and Metabolomics, Pomeranian Medical University in Szczecin, Broniewskiego Str. 24,

71-460 Szczecin, Poland

2 Independent Provincial Public Hospital Complex in Szczecin Zdunowo, Szczecin, Poland the prevalence of non-infectious chronic diseases, including type 2 diabetes (DMII) and cardiovascular diseases [3]. Adipose tissue secretes a number of bioactive substanceshormones and cytokines - called adipokines, which cause systemic chronic inflammation expressed by disorders in the functioning of specific organs, leading to the development of numerous chronic illnesses [4, 5]. Non-alcoholic fatty liver disease, type 2 diabetes, sleep apnea, neoplasms (referring to breasts, ovaries, prostate, liver, gallbladder, kidneys, and colon), and movement system dysfunctions are just some of those issues [2,6]. Obesity is associated with the increased concentration of free fatty acids and proinflammatory factors (such as interleukin (IL) $1 \beta$, IL-6, tumor necrosis factor $\alpha$, and monocyte chemotactic protein-1), as well as of immune cells and their infiltration into the place of inflammation [7]. Obesity facilitates the development of atherogenic dyslipidemia, which is characterized by an increased level of lowdensity lipoproteins (LDL), as well as a decreased level of high-density lipoproteins (HDL) [8]. Dyslipidemia and chronic inflammation lead to the dysfunction of blood vessels, including the formation of atherosclerosis and the disorders of fibrinolysis, both of which increase the risk of developing cardiovascular diseases, including a stroke and the venous thromboembolic disease [9]. In the context of cardiovascular 
diseases, homocysteine (Hcy) is an important indicator. It is an independent risk factor of their development and a marker of the amplification of atherosclerotic changes [10].

In the case of severely obese patients, the surgical treatment of obesity is considered to be the most effective method of reducing body mass [11]. Bariatric surgery is a method of treatment that can be used in the case of patients with $\mathrm{BMI} \geq 35 \mathrm{~kg} / \mathrm{m}^{2}$ and with coexisting complications of obesity, such as arterial hypertension, or with BMI $\geq 40 \mathrm{~kg} / \mathrm{m}^{2}$ [12]. To be considered for surgery, patients had to fail to lose weight or to maintain long-term weight loss, despite appropriate surgical and/or non-surgical comprehensive medical care [12]. In traditional classification, surgical methods have been categorized into 3 main groups: restrictive (which limit the size of the stomach), malabsorptive (which shorten intestinal transit, leading to the reduction of the processes of digestion and absorption), and hybrid (which combine the elements of two previous methods) [13]. Nowadays, this traditional classification has become less functional because of necessity to better understanding the metabolic effects of various bariatric operations, e.g., incretin effects $[14,15]$. At this moment, there is insufficient evidence-based data to suggest how to assign a patient to a specific bariatric procedure with no evidence in favor of any particular procedure. A bariatric surgeon decides about the choice of the surgical method. Some preoperative factors that can influence the choice of the type of surgery are body mass index, age, diabetes mellitus and its duration, and gastroesophageal reflux disease [12]. Currently, the most commonly used methods include restrictive methods — sleeve gastrectomy (SG) — and hybrid methods-Roux-en-Y gastric bypass (RYGB) [16].

The aim of the article was to revise current literature which concerns the risk of high serum homocysteine level in patients after bariatric surgery due to the deficiency of the main methionine metabolism cofactors.

\section{Homocysteine}

Homocysteine is an amino acid that includes thiol groups in its particles. It is formed in all cells of the organism as a result of the metabolism of one of the exogenic amino acids-methionine. The necessary cofactors for the biochemical course of changes of Hcy include folates and vitamin B12 (the methylation process of Hcy into methionine), vitamin B6 (the catabolism of Hcy into cysteine) [17], vitamin B2 (the process of forming 5-methyltetrahydrofolate by means of 5,10methylenetetrahydrofolate reductase), betaine (the remethylation process) [18], and magnesium and lithium (adenosine triphosphate is present in the form of a complex with magnesium ions and lithium ions; this compound, which is high in energy, participates in the transformation of methionine into S-adenosylmethionine via methionine adenosyltransferase) [19-21].

In the serum, Hcy is present mainly in a form that is coupled with albumins and, to a significantly smaller degree, in the form of Hcy or Hcy disulfides coupled with other thiols, e.g., cysteine [22]. Three processes occur as part of Hcy metabolism [22-24]:

1. The remethylation of Hcy into methionine with the use of methionine synthase. In this process, vitamin B12 is the cofactor, whereas the donor of the methyl group is 5methyltetrahydrofolate, which is formed in a reaction catalyzed by 5,10-methylenetetrahydrofolate reductase (MTHFR).

2. Transsulfuration. During which, in the reaction catalyzed by cystathionine $\beta$-synthase, Hcy combines with serine to form cystathionine. The coenzyme in this process is the active form of pyridoxine-pyridoxal phosphate. The acquired cystathionine breaks down and together with $\gamma$ cystathionase forms $\alpha$-ketobutyrate and cysteine. Then, the latter is broken down into taurine or it participates in the synthesis of glutathione.

3. The remethylation of Hcy into methionine via betainehomocysteine methyltransferase (BHMT) in some tissues, i.e., the kidneys and the liver. In this process, betaine is used as the donor of the methyl group, and the products of the reaction are dimethylglycine and methionine.

In physiological conditions, when vitamin B is properly supplied in the diet, the concentration of Hcy in the blood should be correct. An increase in the concentration of Hcy can often be the result of deficiencies of vitamins B6, B12, and folate [25]. Hyperhomocysteinemia is a state defined as an increase in the level of Hcy in the blood beyond $15 \mu \mathrm{mol} / \mathrm{L}$. The values $16-30 \mu \mathrm{mol} / \mathrm{L}, 31-100 \mu \mathrm{mol} / \mathrm{L}$, and $>100 \mu \mathrm{mol} / \mathrm{L}$ confirm the presence of mild, moderate, or severe hyperhomocysteinemia, respectively [26]. Apart from an improperly balanced diet, the increase of the level of Hcy in blood can be the result of diseases of such organs as the kidneys or the thyroid, as well as other factors, such as neoplasms, psoriasis, diabetes, the use of some drugs (e.g., metformin), alcohol consumption, smoking, elderly age, menopause, achlorhydria with a low level of Castle's external factor, intestinal inflammatory diseases, and surgeries of the digestive tract (e.g., bariatric surgeries) $[27,28]$. The factors that contribute to the increase of the level of homocysteine in blood also include gene polymorphisms, mainly in reference to MTHFR - the key enzyme of the folate cycle. The most common of these polymorphisms, and therefore the ones that are associated with reduced enzymatic activity and an increased risk of hyperhomocysteinemia, are [29-31]: 
- Polymorphism $677 \mathrm{C}>\mathrm{T}$, which is responsible for the transformation of cytosine into thymine, so at the same time for the replacement of alanine with valine in the catalytic area of the enzyme (position 222);

- Polymorphism $1298 \mathrm{~A}>\mathrm{C}$, which is the effect of substituting adenine with cytosine in exon 7, resulting in the conversion of glutamine into alanine (position 429).

The 677TT variant is associated with the reduction of the activity of MTHFR by about $70 \%$, whereas 677 CT by $35 \%$ in reference to the wild-type homozygote $677 \mathrm{CC}$ (100\% enzyme activity) [29]. The coexistence of the variant 677TT/1298AA and $677 \mathrm{CT} / 1298 \mathrm{AC}$ reduces the activity of MTHFR by 60 $70 \%$ and $40-50 \%$, respectively $[31,32]$ (Fig. 1).

\section{The Clinical Implications of Hyperhomocysteinemia}

An increased level of homocysteine in blood correlates with the existence of specific pathological units, such as cardiovascular diseases, atherosclerosis, a stroke, depression, Alzheimer's disease, and osteoporosis [10, 34-36]. This results from the fact that Hcy undergoes autoxidation, which leads to the formation of biologically active substances that participate in signaling pathways associated with increased cell toxicity, facilitating apoptosis, necrosis, the formation of blood clots, and the amplification of oxidative stress [18, 37, 38]. The autoxidation of Hcy thiol groups results in the formation of reactive oxygen species (ROS), i.e., hydrogen peroxide. Additionally, by reducing the activity of glutathione peroxidase and the redox potential, Hcy amplifies the effects of ROS. In consequence, lipids, proteins, carbohydrates, and nucleic acids undergo oxidation, leading to endothelium dysfunctions, the damaging of blood vessel walls, the activation of platelets, and the formation of blood clots [39-41]. Hyperhomocysteinemia is also toxic for neurons and glial cells $[39,42]$. Among other reasons, the toxicity is a result of the intracellular mobilization of $\mathrm{Ca}^{2+}$ and oxidative stress within the endoplasmic reticulum, leading to apoptosis, the rebuilding of extracellular matrix in the brain, and endothelium dysfunctions [39, 43]. Furthermore, free oxygen radicals have the ability to induce the activity of the NR1 subunit of the NMDA receptor (N-methyl-D-aspartate), which leads to its increased sensitivity to stimulating amino acids (glycine, serine, and glutamate), resulting in disorders in the integrity of the blood-brain barrier [38, 44].

\section{The Perioperative Concentration of Vitamins B6, B12, and Folate}

Bariatric surgery is considered to be an effective way of treating patients with severe obesity, which leads to the reduction of body mass, coexisting illnesses, and mortality $[45,46]$. However, this method is not free from complications. The most common ones (moderately long and long term) include micronutrient deficiencies, which result from the limited

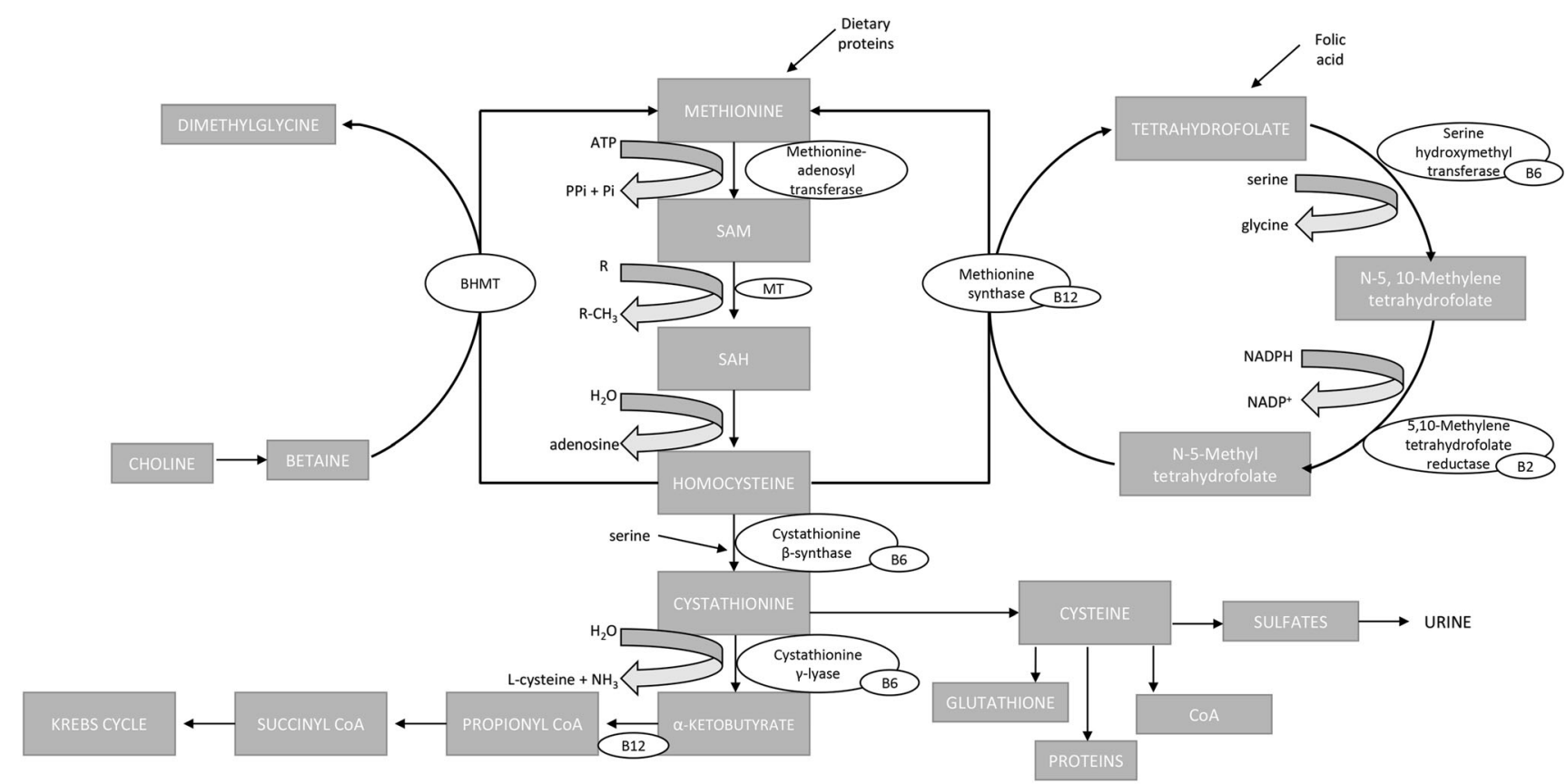

$\mathrm{R}-\mathrm{CH}_{3}$ methylated product; $\mathrm{R}$ demethylated substrate; SAM S-adenosylmethionine; SAH S-adenosylhomocysteine; MT methyltransferases; BHMT betaine:homocysteine methyltransferase; B2, B6, B12 vitamins B2, B6, B12; ATP adenosine triphosphate; PPi pyrophosphate; Pi orthophosphate; CoA coenzyme A

Fig. 1 Scheme of methionine metabolism [18, 33] 
consumption of foods and limited absorption as well as from the combination of the two [47-49]. Obese patients, despite high-energy consumption in general, are characterized by the presence of vitamin and mineral deficiencies before the surgery, which is associated with the low quality of the food they consume [50].

In the study by Sánchez et al. [51], the authors showed that despite a high-caloric diet $(2801 \pm 970 \mathrm{kcal} /$ day $), 66 \%$ of female patients qualified for RYGB or SG surgeries expressed a deficiency of at least a single micronutrient. Vitamin B12 deficiency occurred in $10.6 \%$ of women, but no patient experienced folic acids deficiencies.

Also, in another study [52], nutritional deficiencies were common in bariatric candidates (RYGB and SG), with vitamin B6 deficiency (28\%), followed by folate (26.8\%) and vitamin B12 (3.3\%). One year after surgery, folate deficiency was noticed in 4\% RYGB and $10.8 \%$ SG patients. Authors noticed that despite post-operative routine multivitamin supplementation, the prevalence of vitamin B12 deficiency increased remarkably in the RYGB group (16\%) 12 months post-operatively. Additionally, pre-operative vitamin B12 level was independently associated with post-operative decrease, and deficiency of vitamin B6 before surgery was a predictor for deficiency 1 year after surgery.

Interestingly, Johnson et al. [53] observed that less than 2\% of vitamin B12 deficiencies and no folate deficiencies occurred in both SG and RYGB patients after 1 year post-operatively. In turn, during a mean follow-up period of 4 years post-RYGB or post-SG [54], patients were identified with vitamin B12 and folate deficiencies. Vitamin B12 deficiency was significantly higher in patients with RYGB compared with SG (42.1\% versus 5\%). The authors stated that this difference may result from the fact that SG, being associated with less malabsorption, may have a more favorable effect on the metabolism of vitamin B12 compared with RYGB.

In a different study [55] conducted on patients who underwent SG, $24 \%$ of these patients experienced a decreased concentration of folates in blood, whereas $11.5 \%$ had a deficiency of vitamin B12. Interestingly, $21 \%$ of the patients had excess levels of vitamin B6, whereas only $3 \%$ experienced its deficiency. A year after the surgery, folic acid deficiency was reported in $12.5 \%$ of the patients, vitamin B12 deficiency in $11.5 \%$, and vitamin B6 deficiency in $4 \%$. The presence of hypervitaminosis of pyridoxine after surgery doubled, achieving $47.5 \%$. Even in another study [56], authors noticed that throughout the whole population of the study, only one case of vitamin B6 deficiency was identified before surgery. On the contrary, excess data was the most remarkable (24.1\%). Additionally, only one case of vitamin B6 deficiency was identified throughout the post-operative period. The result is in line with the findings of Aarts et al. [57], Moizé et al. [58], and Gehrer et al. [59] who reported no cases of vitamin B6 deficiency 1,3 , and 5 years post-operatively. In contrast, excess level of pyridoxine was noticed in $38.7 \%, 42.6 \%$, and $23.3 \% 1,3$, and 5 year post-operatively, respectively [56]. This effect might have been a result of the use of multivitamin products that included high doses of vitamin B6 in their content.

In the study by Toh et al. [60], it was observed that the preoperative deficiency of vitamin B12 amplified after RYGB (from 1 to $11 \%$ ). In the case of SG, the deficiency of this vitamin decreased from 4 to $0 \%$. RYGB surgery, because it belongs to hybrid methods, is associated with the increased risk of experiencing vitamin B12 deficits. Several factors contribute to this phenomenon [61-66]:

- The development of intolerance and the limited consumption of products that are a source of wholesome animal protein (mainly meat);

- The decreased concentration of hydrochloric acid and pepsin in the stomach, which are necessary for the separation of vitamin B12 from food proteins;

- The bypass of the duodenum and the shortened contact of nutrients with pancreatic juice which can weaken the hydrolysis of cobalophilin and, in consequence, the release of vitamin B12;

- The decreased availability of the external factor, which is necessary for the absorption of vitamin B12;

- Bacterial overgrowth in the section of the digestive tract excluded from the passage.

In the same study [60], the deficiency of folates in patients (who underwent SG or RYGB surgeries) was at $0 \%$ before and after the surgical intervention. However, the authors labeled one more parameter important in the context of supplying the organism with folates - the amount of folates in red blood cells (RBC folate). This value is a more precise predictor of the long-term status of folates in the organism, whereas the measurement of folate content in the serum may be distorted by the recent consumption of food [67]. It has been observed that low folate content in red blood cells occurred in $1 \%$ of patients before RYGB and in $12 \%$ after the surgery, whereas the numbers for SG were $7 \%$ before and $0 \%$ after the treatment [60]. The aforementioned changes of vitamin B concentrations may have been reflected by the hyperhomocysteinemia observed by the authors. Its frequency of occurrence increased from 5 to $24 \% 1$ year after RYGB and from 18 to $25 \%$ a year after SG. Importantly, the recommendation for all patients after surgery was multivitamin supplementation, and patients after RYGB were additionally recommended to take $1500 \mathrm{mg}$ of calcium citrate and $1000 \mu \mathrm{g}$ of vitamin B12 every 3 to 6 months, administered by intramuscular injection [60]. This fact suggests that apart from the concentration of $\mathrm{B}$ vitamins in blood, there are also other factors that are responsible for the presence of hyperhomocysteinemia after surgery. 


\section{The Concentration of Homocysteine in the Blood of Bariatric Patients}

The influence of bariatric surgeries on the concentration of Hcy in the blood of patients is not clear. Some studies did not report changes (RYGB) [68] in the concentration of Hcy after surgery, whereas other works indicated either its decreased (RYGB and an adjustable gastric band) [69] or increased (RYGB and SG) [60] concentration in blood.

In the study conducted by Sledzinski et al. [70], the authors reported a significantly higher concentration of Hcy in the blood of patients qualified for bariatric operation than in patients with correct BMI $\left(18.5-24.9 \mathrm{~kg} / \mathrm{m}^{2}\right)$. Six months after vertical banded gastroplasty (VBG), the concentration of Hcy in blood did not decrease despite supplementation with vitamins B6, B12, and folate.

In a different study [33], in patients who were 6 months after VBG, there were no significant changes in the concentration of Hcy in blood despite the regular supplementation with vitamins B12 $(1000 \mu \mathrm{g} / \mathrm{month}$, administered by intramuscular injection), B6 $(5 \mathrm{mg} /$ day, administered orally), and folate $(0.4 \mathrm{mg} / \mathrm{day}$, administered orally). The authors demonstrated that the concentration of cobalamin and folates in blood did not change significantly, which results from the satisfactory supplementation of these vitamins after the surgical intervention. At the same time, the lack of influence of vitamins on the decrease of the concentration of Hcy in blood (before the intervention-15 \pm $1.5 \mu \mathrm{mol} / \mathrm{L}$; after the intervention $-14 \pm 1.6 \mu \mathrm{mol} / \mathrm{L}$ ) may point to the presence of other indicators influencing the level of Hcy in this group of patients. It has been observed that in post-surgery patients, the concentration of betaine (the donor of methyl residue from choline) in serum was lower than in the control group (consisting of healthy patients with correct BMI, $18.5-24.9 \mathrm{~kg} / \mathrm{m}^{2}$ ) (Fig. 1). Furthermore, after the surgery, a significant decrease in the ratio between betaine and homocysteine was observed in comparison with pre-surgery values. The authors of the study suggest that the presence of hyperhomocysteinemia after the surgery may be a result of the decreased concentration of betaine in blood after the intervention. Decreased betaine levels might have been caused by the significant reduction of the consumed food, as well as the changes in the functioning of kidneys, which lead to increased betaine loss with urine.

Interestingly, in a study by Ledoux et al. [71], scientists did not observe any correlations between the concentration of B12 and Hcy in patients before and 6 months after gastric bypass surgery. However, it was demonstrated that the concentration of Hcy was inversely proportional to the concentration of folates and the clearance of creatinine and directly proportional to the concentration of creatinine in patients before and after the surgery. The strong correlation between Hcy and creatinine and the clearance of creatinine suggests a strong influence of kidneys on the metabolism of Hcy [72, 73].
Furthermore, it is worth mentioning that the level of Hcy after gastric bypass surgery was significantly lower in patients receiving supplementation with a large dose of folic acid$0.8 \mathrm{mg} /$ day in comparison with patients receiving only $0.2 \mathrm{mg} /$ day [71].

In a different study [74], there was an increase in the concentration of Hcy in blood 2 and 6 months $(12.9 \mu \mathrm{mol} / \mathrm{L}$ and $13.4 \mu \mathrm{mol} / \mathrm{L}$, respectively) after the RYGB and SG surgeries in comparison with the initial values $(11.5 \mu \mathrm{mol} / \mathrm{L})$ despite supplementation with $5 \mu \mathrm{g}$ of vitamin B12 and $400 \mu \mathrm{g}$ of folic acid. Furthermore, scientists reported a significant decrease in the concentration of vitamin B12 (before the intervention $294 \mathrm{pmol} / \mathrm{L}, 2$ months after the intervention $276 \mathrm{pmol} / \mathrm{L}$, 6 months after the intervention $211 \mathrm{pmol} / \mathrm{L}$ ) and holotranscobalamin (holo-TC) (before the intervention $56 \mathrm{pmol} / \mathrm{L}, 2$ months after the intervention $54 \mathrm{pmol} / \mathrm{L}, 6$ months after the intervention $30 \mathrm{pmol} / \mathrm{L}$ ), as well as a significant increase in the concentration of methylmalonic acid (MMA) (before the intervention $0.18 \mu \mathrm{mol} / \mathrm{L}, 2$ months after the intervention $0.19 \mu \mathrm{mol} / \mathrm{L}, 6$ months after the intervention $0.22 \mu \mathrm{mol} / \mathrm{L}$ ) in the blood of the studied patients [74]. Holo-TC is a form of vitamin $\mathrm{B} 12$ bioavailable to the organism. This is why it can serve as a bioactive vitamin content marker in blood. MMA accumulates in the organism as a result of the deficiency of $\mathrm{B} 12$, which leads to the inactivation of methylmalonyl-CoA mutase, resulting in the accumulation of methylmalonyl-CoA, which enters the bloodstream as free MMA. This is why both of the aforementioned markers can be considered early indicators of cobalamin in the organism [75] (Table 1).

\section{Summary}

The review of literature suggests that bariatric surgeries, via the deficiencies of methionine metabolism cofactors, can significantly influence homocysteine metabolism. The factors that are important in this process include not only balanced nutrition and the right set of supplements but also the type of the conducted surgery. Interventions which lead to the reduction of the processes of digestion and absorption have a higher risk of hyperhomocysteinemia development than methods which limit only the size of the stomach, e.g., due to the increased risk of developing vitamin $\mathrm{B} 12$ deficiencies. It is worth to consider MMA and holo-TC assays as early markers of vitamin B12 deficiency, especially in the case of patients with hyperhomocysteinemia and B12 levels within norms. Post-surgical hyperhomocysteinemia can also be supported by the reduced concentration of betaine and changes in the functioning of kidneys. Further, separate but important factors are the mutations of the MTHFR gene, which considerably slow down the metabolism of the folic cycle and can be one of the reasons for the persistent high concentration of Hcy in blood after surgery despite supplementation with B-group vitamins. 


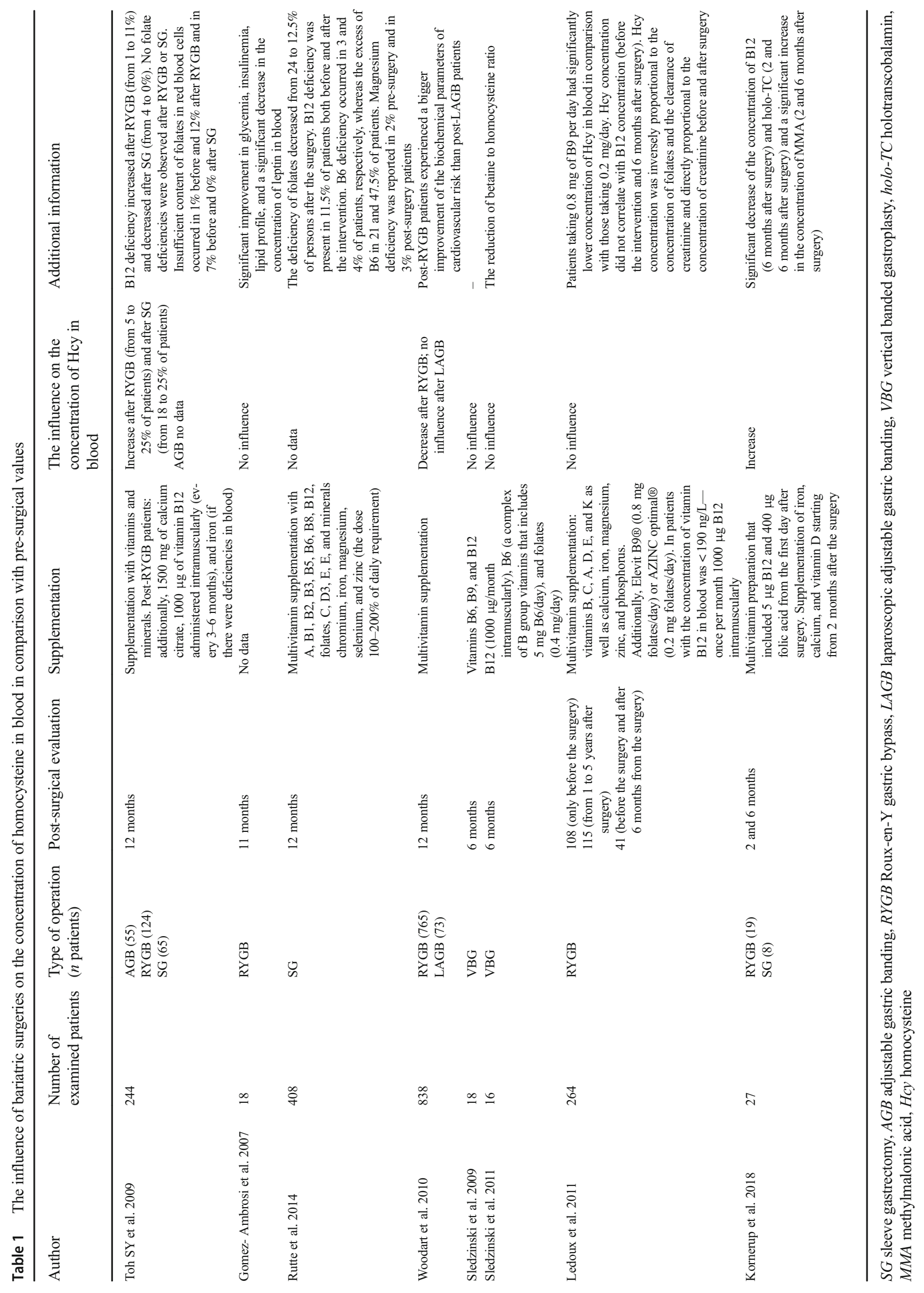




\section{Conclusion}

It is worth to consider a routine examination of the homocysteine level before and after bariatric surgery. It seems to be important especially in people with diseases that co-occur with elevated homocysteine levels in the blood, such as diabetes, atherosclerosis, and depression. If, despite the of routine multivitamin supplementation, homocysteine levels remain elevated, it is worth considering the administration of the methylated form of folic acid and vitamin B12. Undoubtedly, it is necessary to conduct further research in order to identify the factors that are responsible for the persistence of hyperhomocysteinemia in patients qualified for the surgical treatment of obesity.

Funding The project is financed from the program of the Minister of Science and Higher Education under the name "Regional Initiative of Excellence" in 2019-2022 project number 002/RID/2018/19.

\section{Compliance with Ethical Standards}

Conflict of Interest The authors declare that they have no conflict of interest.

Statement of Informed Consent Informed consent was obtained from all individual participants included in the study.

\section{Statement of Human and Animal Rights/Ethical Approval Not applicable.}

Open Access This article is distributed under the terms of the Creative Commons Attribution 4.0 International License (http:// creativecommons.org/licenses/by/4.0/), which permits unrestricted use, distribution, and reproduction in any medium, provided you give appropriate credit to the original author(s) and the source, provide a link to the Creative Commons license, and indicate if changes were made.

\section{References}

1. Fruh SM. Obesity: risk factors, complications and strategies for sustainable long-term weight management. J Am Assoc Nurse Pract. 2017;29(1):3-14.

2. World Health Organization http://www.who.int/en/news-room/factsheets/detail/obesity-and-overweight (30.10.2018)

3. Wang YC, McPherson K, Marsh T, et al. Health and economic burden of the projected obesity trends in the USA and the UK. Lancet. 2011;378:815-25.

4. Halberg N, Wernstedt-Asterholm I, Scherer PE. The adipocyte as an endocrne cell. Endocrinol Metab Clin N Am. 2008;37:753-68.

5. Alexopoulos N, Katritsis D, Raggi P. Viscelar adipose tissue as a source of inflammation and promoter of atherosclerosis. Atherosclerosis. 2014;233:104-12.

6. Guh DP, Zhang W, Bansback N, et al. The incidence of comorbidities related to obesity and overweight: a systematic review and metaanalysis. BMC Public Health. 2009;9:88.

7. Hursting SD, Dunlap SM. Obesity, metabolic dysregulation and cancer. A growing concern and an inflammatory (and microenvironmental) issue. Ann N Y Acad Sci. 2012;1271:82-7.
8. Musunuru K. Atherogenic dyslipidemia: cardiovascular risk and dietary intervention. Lipids. 2010;45(10):907-14.

9. Blohkin IO, Lentz SR. Mechanism of thrombosis in obesity. Curr Opin Hematol. 2013;20(5):437-44.

10. Sreckovic B, Dimitrijevic Sreckovic V, Soldatovic I, et al. Homocysteine is a marker for metabolic syndrome and atherosclerosis. Diabetes Metab Syndr. 2017;11(3):179-82.

11. Hady HR, Dadan J, Iwacewicz P. Evaluation of the efficiency of chosen methods of surgical treatment of pathological obesity in our own material. Wideochir Inne Tech Maloinwazyjne. 2008;3(2):39-44.

12. Fried M, Yumuk V, Oppert JM, et al. Interdisciplinary European guidelines on metabolic and bariatric surgery. Obes Surg. 2014;24(1):42-55.

13. Shahzeer K, Stoklossa CJ, Sharma A, et al. Bariatric surgery. Can Fam Physician. 2010;56(9):873-9.

14. Mechanick JI, Youdim A, Jones DB, et al. AACE/TOS/ASMBS Guidelines. Clinical practice guidelines for perioperative nutritional, metabolic, and nonsurgical support of the bariatric surgery patients 2013 update: cosponsored by American Association of Clinical Endocrinologists, The Obesity Society and American Society for Metabolic \& Bariatric Surgery. Surg Obes Relat Dis. 2013;9(2):159-91.

15. Laferrère B. Bariatric surgery and obesity: influence on the incretins. Int J Obes Suppl. 2016;6(1):32-6.

16. Kizy S, Jahansouz C, Downey MC, et al. National trends in bariatric surgery 2012-2015: demographics, procedure selection, readmissions, and cost. Obes Surg. 2017;27(11):2933-9.

17. Tzotzas T, Evangelou P, Kiortsis DN. Obesity, weight loss and conditional cardiovascular risk factors. Obes Rev. 2011;12(5):282-90.

18. Škovierová H, Vidomanová E, Mahmood S, et al. The molecular and cellular effect of homocysteine metabolism imbalance on human health. Int J Mol Sci. 2016;17(10): 1733.

19. Stipanuk MH. Sulfur amino acid metabolism: pathways for production and removal of homocysteine and cysteine. Annu Rev Nutr. 2004;24:539-77.

20. Ko YH, Hong S, Pedersen PL. Chemical mechanism of ATP synthase. Magnesium plays a pivotal role in formation of the transition state where ATP is synthesized from ADP and inorganic phosphate. J Biol Chem. 1999;174(41):28853-6.

21. Briggs KT, Giulian GG, Li G, et al. A molecular model for lithium's bioactive form. Biophys J. 2016;111(2):294-300.

22. Maron BA, Loscalzo J. Should hyperhomocysteinemia be treated in patients with atherosclerotic disease? Curr Atheroscler Rep. 2007;9:375-83.

23. Finkelstein JD. Methionine metabolism in mammals. J Nutr Biochem. 1990;1:228-37.

24. Simile MM, Latte G, Feo CF, et al. Alterations of methionine metabolism in hepatocarcinogenesis: the emergent role of glycine $\mathrm{N}$ methyltransferase in liver injury. Ann Gastroenterol. 2018;31(5): 552-60.

25. Curro M, Gugliandolo A, Gangemi C, et al. Toxic effects of mildl elevated homocysteine concentrations in neuronal-like cells. Neurochem Res. 2014;30:1485-95.

26. Ganguly P, Alam SF. Role of homocysteine in the development of cardiovascular disease. Nutr J. 2015;14:6.

27. Faeh D, Chiolero A, Paccaud F. Homocysteine as a risk factor for cardiovascular disease: should we (still) worry about it? Swiss Med Wkly. 2006;136:745-56.

28. Andrès E, Affenberger S, Vinzio S, et al. Food-cobalamin malabsorption in elderly patients: clinical manifestations and treatment. Am J Med. 2005;118:1154-9.

29. Frosst $\mathrm{P}, \mathrm{Blom} \mathrm{HJ}$, Milos R, et al. A candidate genetic risk factor for vascular disease: a common mutation in methylenotetrahydrofolate reductase. Nat Genet. 1995;10(1):111-3.

30. Rozen R. Molecular genetics of methylenotetrahydrofolate reductase deficiency. J Inher Metab Dis. 1996;19:589-94. 
31. Van der Put NM, Gabreëls F, Stevens EM, et al. A second common mutation in the methylenotetrahydrofolate reductase gene: an additional risk factor for neural-tube defects? Am J Hum Genet. 1998;62(5):1044-51.

32. Weisberg I, Tran P, Christensen B, et al. A second polymorphism in methylenotetrahydrofolate reductase (MTHFR) associated with decreased enzyme activity. Mol Genet Metab. 1998;64(3):169-72.

33. Sledzinski T, Goyke E, Smolenski RT, et al. Decreased serum betaine concentrations in patients after bariatric surgery. Obes Surg. 2011;21:1634-9.

34. Bhatia P, Singh N. Homocysteine excess: delineating the possible mechanism of neurotoxicity and depression. Fundam Clin Pharmacol. 2015;29(6):522-8.

35. Farina N, Jernerén F, Turner C, et al. Homocysteine concentrations in the cognitive progression of Alzheimer's disease. Exp Gerontol. 2017;99:146-50.

36. Saito M, Marumo K. The effects of homocysteine on the skeleton. Curr Osteoporos Rep. 2018;16(5):554-60.

37. Lubos E, Loscalzo J, Handy DE. Homocysteine and glutathione peroxidase-1. Antioxid Redox Signal. 2007;9:1923-40.

38. Boldyrev A, Bryushkova E, Mashkina A, et al. Why is homocysteine toxic for the nervous and immune systems? Curr Aging Sci. 2013;6(1):29-36.

39. Petras M, Tatarkova Z, Kovalska M, et al. Hyperhomocysteinemia as a risk factor for neuronal system disorder. J Physiol Pharmacol. 2014;65:15-23.

40. Faraci FM, Lentz SR. Hyoerhomocysteinemia, oxidative stress and cerebral vascular dysfunction. Stroke. 2004;35:345-7.

41. Wu X, Zhang L, Miao Y, et al. Homocysteine causes vascular endothelial dysfunction by disrupting endoplasmic reticulum redox homeostasis. Rdox Biol. 2019;20:45-59.

42. Škovierová H, Mahmood S, Blahovcová E, et al. Effect of homocysteine on survival of human glial cells. Physiol Res. 2015;64:747-54.

43. Lehotsky J, Petras M, Kovalska M, et al. Mechanisms involved in the ischemic tolerance in brain: effect of homocysteine. Cell Mol Neurobiol. 2015;35:7-15.

44. Betzen $\mathrm{C}$, White $\mathrm{R}$, Zehender CM, et al. Oxidative stress upregulates the NMDA receptor on cerebrovascular endothelium. Free Radic Biol Med. 2009;47:1212-20.

45. Sjöström L, Lindroos AK, Peltonen M, et al. Lifestyle, diabetes and cardiovascular risk factors 10 years after bariatric surgery. N Engl J Med. 2004;351(26):2683-93.

46. Sjöström L, Narbro K, Sjöström CD, et al. Effects of bariatric surgery on mortality in Swedish obese subjects. N Engl J Med. 2007;357(8):741-52.

47. Shikora SA, Kim JJ, Tarnoff ME. Nutrition and gastrointestinal complications of bariatric surgery. Nutr Clin Pract. 2007;22(1):29-40.

48. Poitou BC, Ciangura C, Coupaye M, et al. Nutritional deficiency after gastric bypass: diagnosis, prevention and treatment. Diabetes Metab. 2007;33(1):13-24.

49. Xanthakos SA. Nutritional deficiencies in obesity and after bariatric surgery. Pediatr Clin N Am. 2009;56(5):1105-21.

50. Roust LR, DiBaise JK. Nutrient deficiencies prior to bariatric surgery. Curr Opin Clin Nutr Metab Care. 2017;20:138-44.

51. Sánchez A, Rojas P, Basfi-fer K, et al. Micronutrient deficiencies in morbidly obese women prior to bariatric surgery. Obes Surg. 2016;26:361-8.

52. Guan B, Yang J, Chen Y, et al. Nutritional deficiencies in Chinese patients undergoing gastric bypass and sleeve gastrectomy: prevalence and predictors. Obes Surg. 2018;28(9):2727-36.

53. Johnson LM, Ikramuddin S, Leslie DB, et al. Analysis of vitamin levels and deficiencies in bariatric surgery patients: a singleinstitutional analysis. Surg Obes Relat Dis. 2019;

54. Alexandrou A, Armeni E, Kouskouni E, et al. Cross-sectional longterm micronutrient deficiencies after sleeve gastrectomy versus
Roux-en-Y gastric bypass: a pilot study. Surg Obes Relat Dis. 2014;10(2):262-8.

55. Rutte PWJ, Aarts EO, Smulders JF, et al. Nutrient deficiences before and after sleeve gastrectomy. Obes Surg. 2014;24:1639-46.

56. Al-Mutawa A, Al-Sabah S, Anderson AK, et al. Evaluation of nutritional status post laparoscopic sleeve gastrectomy -5 -year outcomes. Obes Surg. 2018;28:1473-83.

57. Aarts E, Janssen I, Berends F. The gastric sleeve: losing weight as fast as micronutrients? Obes Surg. 2011;21(2):207-11.

58. Moizé V, Deulofeu R, Torres F, et al. Nutritional intake and prevalence of nutritional deficiences prior to surgery in a Spanish morbidly obese population. Obes Surg. 2011;21(9):1382-8.

59. Gehrer S, Kern B, Peters T, et al. Fewer nutrient deficiencies after laparoscopic sleeve gastrectomy (LSG) than after laparoscopic Roux-en-Y gastric bypass (LRYGB) - a prospective study. Obes Surg. 2010;20(4):447-53.

60. Toh SY, Zarshenas N, Jorgensen J. Prevalence of nutrient deficiencies in bariatric patients. Nutrition. 2009;25(11-12):1150-6.

61. Vargas-Ruiz AG, Hernandez-Rivera G, Herrera MF. Prevalence of iron, folate, and vitamin B12 deficiency anemia after laparoscopic Roux-en-Y gastric bypass. Obes Surg. 2008;18:288-93.

62. Buchwald $\mathrm{H}$, Avidor Y, Braunwald E, et al. Bariatric surgery: a systemic review and meta-analysis. JAMA. 2004;292:1724-37.

63. Maggard MA, Shugarman LR, Suttorp M, et al. Meta-analysis: surgical treatment of obesity. Ann Intern Med. 2005;142:547-59.

64. Kushner RF. Micronutrient deficiencies and bariatric surgery. Curr Opin Endocrinol Diabetes. 2006;13:405-11.

65. Hunt A, Harrington D, Robinson S. Vitamin B12 deficiency. BMJ. 2014;349:g5226.

66. Oxentenko AS, Litin SC. Clinical pearls in gastroenterology. Mayo Clin Proc. 2009;84(10):906-11.

67. Piyathilake CJ, Robinson CB, Cornwell P. A practical approach to red blood cell folate analysis. Anal Chem Insights. 2007;2:107-10.

68. Gómez-Ambrosi J, Pastor C, Salvador J, et al. Influence of waist circumference on the metabolic risk associated with impaired fasting glucose: effect of weight loss after gastric bypass. Obes Surg. 2007;17:585-91.

69. Woodard GA, Peraza J, Bravo S, et al. One-year improvements in cardiovascular risk factors: a comparative trial of laparoscopic Roux-en-Y gastric bypass vs adjustable gastric banding. Obes Surg. 2010;20(5):578-82.

70. Sledzinski T, Goyke E, Smolenski RT, et al. Decrease in serum protein carbonyl groups concentration and maintained hyperhomocysteinemia in patients undergoing bariatric surgery. Obes Surg. 2009;19:321-6.

71. Ledoux S, Coupaye M, Bogard C, et al. Determinants of hyperhomocysteinemia after gastric bypass surgery in obese subjects. Obes Surg. 2011;21:78-86.

72. Nouri-Majalan N, Masoumi R, Nafisi R, et al. Relationship between serum homocysteine and other parameters in renal transplant patients. Transplant Proc. 2009;41(7):2826-8.

73. Serpa NA, Bianso Rossi FM, Dal Moro Amarante R, et al. Effect of weight loss after Roux-en-Y gastric bypass on renal function and blood pressure in morbidly obese patients. J Nephrol. 2009;22(5):637-48.

74. Kornerup LS, Hvas CL, Abild CB, et al. Early changes in vitamin B12 uptake and biomarkers status following Roux-en-Y gastric bypass and sleeve gastrectomy. Clin Nutr. 2018:1-6.

75. Hannibal L, Lysne V, Bjørke-Monsen A-L, et al. Biomarkers and algorithms for the diagnosis of vitamin B12 deficiency. Front Mol Biosci. 2016;3:27.

Publisher's Note Springer Nature remains neutral with regard to jurisdictional claims in published maps and institutional affiliations. 